\title{
DENS INVAGINATUS TIPO III: REPORTE DE UN CASO
}

\author{
${ }^{1}$ Lidia Hortensia Morales Amaya, ${ }^{2}$ Patricia Trejo Quiroz, ${ }^{3}$ Daniela Carmona Ruiz, ${ }^{2}$ Claudia De León Torres \\ ${ }^{1}$ Especialista en Odontopediatría DEPeI F. de Odontología U. Autónoma Nacional de México, Ciudad de México (México) \\ ${ }^{2}$ Docente Especialidad en Odontopediatría DEPeI F. de Odontología U. Autónoma Nacional de México, Ciudad de México (México) \\ ${ }^{3}$ Docente de Odontología Preventiva y Salud Pública Bucal F. de Odontología U. Autónoma Nacional de México, Ciudad de México (México) \\ ${ }^{1}$ Especialista en Odontopediatría DEPeI F. de Odontología U. Autónoma Nacional de México, Ciudad de México (México)
}

\section{Autor responsable de correspondencia: Claudia De León T.}

Correo electrónico: cdeleont@gmail.com

\section{RESUMEN}

El dens invaginatus es una alteración del desarrollo que se produce por una invaginación del epitelio interno del órgano del esmalte, dentro de la papila dental, durante los estadios tempranos de la morfodiferenciación, de etiología multifactorial. Se reporta el caso clínico de una paciente de 9 años de edad, en el examen clínico se observó fístula, el incisivo central derecho con macrodoncia y un cíngulo exagerado, la paciente no refería antecedentes de dolor ni de traumatismos. Radiográficamente, encontró una invaginación que atraviesa la raíz formándose dos forámenes apicales, por lo que se diagnosticó como dens invaginatus tipo III, con necrosis pulpar. [Morales LH, Trejo P, Carmona D, De León CS. Dens invaginatus tipo III: reporte de un caso. Ustasalud 2011; 10: 132 - 134]

Palabras clave: Dens invaginatus, Diagnóstico.

\section{DENS INVAGINATUS TYPE III: A CASE REPORT}

\section{ABSTRACT}

Dens invaginatus is a developmental disorder that is caused by an invagination of the inner epithelium of the enamel organ, inside dental papilla, during the early stages of morphodifferentiation of multifactorial etiology. A case of a 9 years-old female patient is presented, during the clinical examination it was observed fistula and the right central incisor with macrodontia, the patient referred no history of pain or trauma. Radiographically, it was observed an invagination that crosses the root forming two apical foramina, so that was diagnosed as type III dens invaginatus with pulp necrosis

Key words: Dens invaginatus, Diagnosis

Recibido para publicación: 24 de agosto de 2011. Aceptado para publicación: 13 de noviembre de 2011

\section{INTRODUCCIÓN}

Los sinónimos utilizados para el dens invaginatus son dens in dente, odontoma invaginado, odontoma gestante dilatado, odontoma compuesto dilatado, inclusión dental, dentoid in dente. El dens invaginatus es una alteración del desarrollo que se produce por una invaginación del epitelio interno del órgano del esmalte, dentro de la papila dental, durante los estadios tempranos de la morfodiferenciación. La corona dental así como las raíces pueden mostrar variaciones en forma y tamaño.,

Radiográficamente, se muestra una invaginación de esmalte y dentina la cual puede extenderse a la cámara pulpar, raíz y en algunas ocasiones al ápice radicular. En la mayoría de los casos esta alteración suele ser asintomática y en muchas ocasiones se encontrará tras un examen radiológico de rutina.

El dens invaginatus fue descrito por primera vez en 1856. En 1873 Mühlreiter, Baume en 1874 y
Tomes en 1897. Posteriormente Busch los describió en 1887. La etiología de esta alteración del desarrollo es controversial y diversas teorías han sido propuestas:

- Presión anormal en el arco dental que resulta en un encorvamiento del órgano del esmalte.

- En 1934, Kronfeld explicó que era resultado de una proliferación rápida y agresiva del epitelio interno del esmalte que invade la papila dental.

- Fischer, en 1936, considera que un proceso infeccioso es el responsable de esta malformación, al igual que Sprawson.

- En 1950 Gustafson y Sundberg consideraron como un probable factor etiológico al trauma.

- En 1957, Oehlers que es una distorsión del órgano del esmalte durante el desarrollo del diente.

- Así también los factores genéticos se asocian desde 1962 (Granhen). Hosey y Bedi en 1996 coinciden con la teoría. 


\section{Clasificación de Oehlers}

La clasificación de Oehlers fue propuesta en 1957 y es la más comúnmente usada. Se describe en relación a la extensión de la invaginación y las clasificó en 3 tipos:

- Tipo I: limitada a la corona sin pasar la unión amelocementaria

- Tipo II: la invaginación va más allá de la unión amelocementaria y puede o no estar comunicada con la pulpa

- Tipo III: penetra a través de la raíz perforando el área apical y muestra un segundo foramen en el área periapical o en el área periodontal (Figura 1).

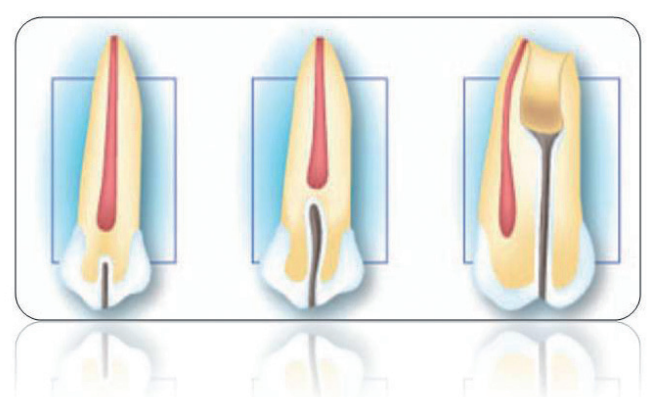

Figura 1. Clasificación de Oehlers

Los dientes más comúnmente afectados son los incisivos laterales inferiores. El diagnóstico en $\mathrm{mu}$ chas ocasiones se realiza a través de un examen radiográfico. Clínicamente, se realiza por una corona morfológicamente alterada, o con un foramen profundo, pero los dientes afectados pueden no mostrar signos clínicos. El dens invaginatus puede estar asociado a otras anomalías dentales, malformaciones e incluso síndromes. ${ }^{3}$ Así también el dens invaginatus radicular es la anomalía dental más rara, existen solo tres casos reportados en la literatura. ${ }^{4}$

\section{Tratamiento}

El tratamiento puede ser preventivo o restaurativo. Los dientes con invaginaciones profundas pueden ser tratados con selladores de fosetas y fisuras antes que se presente algún proceso carioso. Se recomienda revisiones periódicas estrictas.

En el caso de necesitar tratamiento de conductos, la primera opción es la terapia convencional. Este tratamiento puede llegar a presentar complicaciones debido a la forma irregular de los conductos.

Cuando existe necrosis pulpar antes del cierre apical la opción de tratamiento serán los procedimientos de apexificación con hidróxido de calcio. En los casos de fracaso en el tratamiento de con- ductos, deben realizarse procedimientos quirúrgicos, como la obturación retrógrada.

Por último, debe indicarse la extracción sólo en los dientes con severas irregularidades anatómicas que no pueden ser resueltas con tratamientos de conductos o procedimientos quirúrgicos. Así también estará indicada en el caso de dientes supernumerarios que presenten esta malformación o en dientes en los que la morfología de la corona provoque problemas funcionales o estéticos. ${ }^{1,5}$

La importancia clínica de esta malformación radica a una posible afección cariosa a través de la comunicación de la porción invaginada de la superficie lingual del diente con el medio exterior. El esmalte y la dentina pueden encontrarse defectuosos o simplemente estar ausentes, por lo que la pulpa queda expuesta directamente. ${ }^{6}$

\section{REPORTE DE CASO}

Paciente de género femenino de 9 años que acude a la Clínica de Odontopediatría de la División de Estudios de Posgrado e Investigación de la facultad de odontología de la Universidad Nacional Autónoma de México. El motivo principal de la consulta fue revisión periódica, se realizó una exploración clínica y radiográfica.

A la exploración clínica se observó un absceso en la zona anterior superior, el incisivo central derecho presentaba macrodoncia y cíngulo exagerado, que no interfería en la oclusión (Figura 2). Se realizaron pruebas de vitalidad, al frío y al calor, las cuales resultaron negativas, la paciente no presentaba dolor a la percusión horizontal ni apical. Por lo que se da el diagnóstico necrosis pulpar.
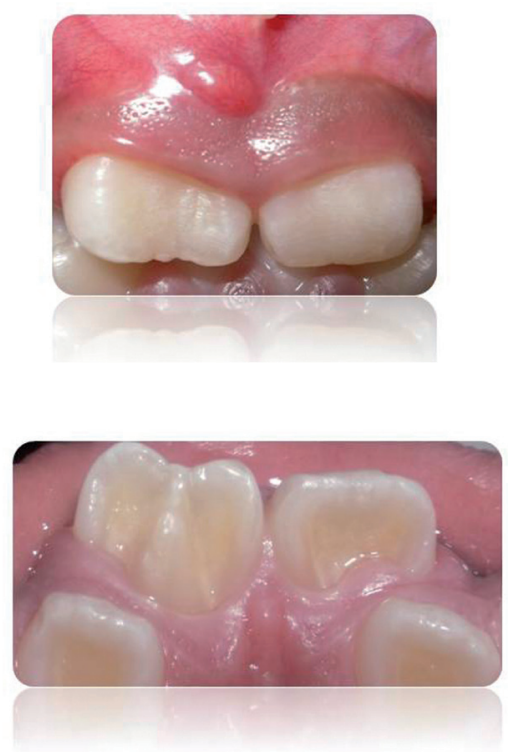

Figura 2. Características clínicas 
Radiográficamente, se pudo observar una invaginación que atravesaba la raíz formándose dos forámenes apicales. Así mismo en la zona periapical se apreció una zona radio-lúcida (Figura 3).

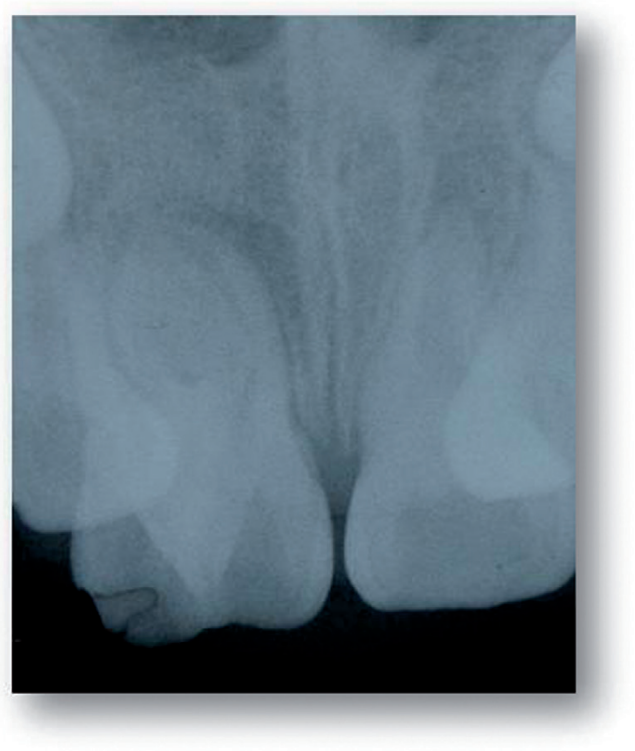

Figura 3. Radiografia que muestra la invaginación a través de la raíz dental

\section{DISCUSIÓN}

El dens invaginatus es una malformación poco común del diente, y muestra un amplio espectro de variaciones morfológicas. El diagnóstico temprano y las características específicas de cada diente serán determinantes para la elección del tratamiento. El tratamiento podría iniciar como preventivo o restaurativo, en donde Rotstein et al. 1987, Hülsmann y Radlanski 1994 recomiendan una restauración de composite bajo un estricto control de revisiones. ${ }^{1}$

Cuando es necesario el tratamiento de conductos, debe tomarse en cuenta las dificultades anatómicas y prever el fracaso en el tratamiento y tener como otra opción el re-tratamiento ya sea quirúrgico o no quirúrgico. La extracción será la última opción en estos casos. Si el diente presenta severas malformaciones, lo cual resulta evidente a la exploración clínica y con el estudio radiográfico de rutina y estas alteraciones morfológicas imposibilitan la realización de algún tratamiento, se deberá proceder a la extracción.

El dens invaginatus es una alteración del desarrollo del diente poco común, por lo que es importante conocer las características principales para poder reconocer y diagnosticar correctamente este tipo de anomalías, por lo que se recomienda como en todos los casos, un examen radiográfico de rutina para detectar cualquier tipo de anomalía

\section{BIBLIOGRAFÍA}

1. Hülsmann M. Dens invaginatus: aetology, classification, prevalence, diagnosis, and treatment considerations. Int End J 1997; 30: 79 - 90

2. Lucas G. Dens invaginatus: tratamiento de un diente permanente joven. Rev ADM 2003; 40: 229 - 232

3. Tiku A, NAdkarni U, Damle SG. Management of two unusual cases of dens invaginatus and talon cusp associated with other dental anomalies. J Indian Soc Pedod Prev Dent 2004; 22: 128 - 133

4. Pandey SC, Pandey RK. Radicular dens invaginatus - a case report. J Indian Soc Pedod Prev Dent 2005; 23: 151 - 152

5. Silberman A, Cohenca N, Simon J. Anatomical redesign for the treatment of dens invaginatus type III with open apexes. J Am Dent Assoc 2006; 137: 180 - 185

6. Pinkham JR, Casamassimo PS, Fields HW, McTigue DJ. Pediatric Dentistry: Infancy Through Adolescence. 3er. Edition, 1999

7. Gupta R, Tewari S. Non surgical management of two unusual cases of dens in dente. J Indian Soc Pedod Prev Dent 2005; 23: 190 - 192

\section{Correos electrónicos de los autores:}

Lidia Hortensia Morales Amaya: lid_daikimyy@hotmail.com Patricia Trejo Quiroz: pichonx3m@gmail.com Daniela Carmona Ruiz: danielacarmona@hotmail.com Claudia Sofia de León Torres: cdeleont@gmail.com 